\title{
Gravidez na adolescência e baixo peso ao nascer: existe associação?
}

\author{
Pregnancy in teenagers and low birthweight infant: is there an association?
}

\begin{abstract}
Almir de Castro Neves Filho ${ }^{1}$, Álvaro Jorge M. Leite ${ }^{2}$, Zenilda Vieira Bruno ${ }^{3}$, José Gomes B. Filho ${ }^{4}$, Cristiana Ferreira da Silva ${ }^{5}$
\end{abstract}
\section{RESUMO}

Objetivo: Verificar a associação entre gravidez na adolescência (dez a 19 anos) e baixo peso ao nascer.

Métodos: Estudo transversal realizado em maternidade terciária entre junho de 2000 a junho de 2001. Por sorteio aleatório simples, foram selecionadas mães adolescentes e não adolescentes entrevistadas no primeiro dia após o parto. Aplicou-se a análise multivariada por meio de regressão logística das variáveis implicadas com o baixo peso ao nascer.

Resultados: Foram selecionadas 539 duplas (mães e seus respectivos recém-nascidos), sendo 331 (61,4\%) mulheres com 20 anos ou mais e 208 (38,5\%) abaixo de 20 anos. Entre as adolescentes, 50 bebês (24\%) tiveram idade gestacional $<37$ semanas, enquanto entre as mães acima de 20 anos, 52 $(15,7 \%)$ eram prematuros (OR 1,58; IC95\% 1,00-2,51). Entre as adolescentes, ocorreram $52(25 \%)$ recém-nascidos com peso $<2500 \mathrm{~g}$ e, entre as adultas, $56(16,9 \%)$ tinham baixo peso (OR 1,64; IC95\% 1,05-2,56). Entre as que realizaram pré-natal adequado, a ocorrência de baixo peso foi de $12,3 \%$ e de $22,1 \%$ no grupo cuja assistência foi inadequada. Nesta casuística, a associação encontrada na análise univariada entre baixo peso e mãe adolescente não se manteve na análise multivariada.

Instituição: Maternidade Escola Assis Chateaubriand (MEAC), Fortaleza, CE, Brasil

${ }^{1}$ Doutorando em Pediatria pela Universidade Federal de São Paulo (Unifesp); Professor Assistente do Departamento de Saúde Materno Infantil da Faculdade de Medicina da Universidade Federal do Ceará (UFC), Fortaleza, CE, Brasil

${ }^{2}$ Doutor em Pediatria pela Unifesp; Professor Adjunto do Departamento de Saúde Materno Infantil da Faculdade de Medicina da UFC, Fortaleza, CE, Brasil

${ }^{3}$ Doutora em Ginecologia pela Universidade Federal do Rio de Janeiro (UFRJ); Professora Associada do Departamento de Saúde Materno Infantil da UFC; Diretora Geral da MEAC, Fortaleza, CE, Brasil

${ }^{4}$ Doutor em Saúde Coletiva pela Universidade Federal da Bahia (UFBA); Professor Adjunto do Departamento de Saúde Comunitária da Faculdade de Medicina da UFC, Fortaleza, CE, Brasil

${ }^{5}$ Doutoranda em Saúde Coletiva pela UFC; Mestre em Saúde Pública pela UFC; Técnica do Núcleo Hospitalar de Epidemiologia do Hospital Municipal de Maracanaú Dr. João Elísio de Holanda, Fortaleza, CE, Brasil
Conclusões: Baixo peso ao nascer em gestações na adolescência não pode ser atribuído isoladamente à idade materna.

Palavras-chave: gravidez na adolescência; recém-nascido de baixo peso; morbidade.

\section{ABSTRACT}

Objective: To study the association between teenage pregnancy (ten to 19 years old) and low birthweight.

Methods: Cross-sectional study carried out at a tertiary center from June 2000 to June 2001. A simple random drawing selected teenagers and adult mothers who were interviewed during the first day after birth. Multivariate analysis using logistic regression of variables related to low birthweight was applied.

Results: 539 pairs (mothers and their newborns) were studied - $331(61.4 \%)$ women with 20 years old or more and $208(38.5 \%)<20$ years old. Among the adolescents, $50(24 \%)$ infants had gestational age $<37$ weeks, whereas among mothers over 20 years old, $52(15.7 \%)$ were preterm (OR 1.58; 95\%CI 1.00-2.51). Among the adolescent and adult women, there were respectively $52(25 \%)$ and 56 (16.9\%) newborns <2500g (OR 1.64; 95\%CI 1.05-2.56). Among those who had adequate prenatal care, low birth

Endereço para correspondência:

Almir de Castro Neves Filho

Rua Papi Júnior, 1.225 - Rodolfo Teófilo

CEP 60970431 - Fortaleza/CE

E-mail: acneves@fortalnet.com.br.

Conflito de interesse: nada a declarar

Recebido em: 25/2/2010

Aprovado em: 21/2/2011 
weight was detected in 12.3 and $22.1 \%$ of mothers with adequate and inadequate prenatal care, respectively. The association found in the univariate analysis between low birth weight and maternal age was not sustained in the multivariate analysis.

Conclusions: Low birth weight in teenage pregnancies can not be attributed only to maternal age.

Key-words: pregnancy in adolescence; infant, low birth weight; morbidity.

\section{Introdução}

A gravidez na adolescência vem despertando crescente interesse e constitui, atualmente, tema de investigações na área de Saúde Pública, que articula conhecimentos das ciências humanas e biológicas. Questões como os riscos perinatais nessas gestações permanecem em aberto e o acervo de publicações sobre o assunto subsidia a análise e o planejamento das estratégias de enfrentamento desse desafio para a área da saúde.

Na maioria dos países da América Latina, as taxas de fecundidade total apresentaram importante declínio nas últimas décadas, contrastando com as taxas de fecundidade na adolescência, cujos valores caíram muito lentamente ou mesmo aumentaram ${ }^{(1-6)}$. No Brasil, o Sistema de Informações sobre Nascidos Vivos (Sinasc) registrou um aumento no número de nascidos vivos de mulheres entre dez e 19 anos de 19,8\% em 1994 para 21,1\% em 2007, representando acréscimo acumulado de 6,8\%. Dados sobre a mortalidade registraram aumento de 6,5\%, entre 1996 e 2007, das mortes causadas diretamente por gravidez, parto, puerpério e suas complicações em adolescentes de 15 a $19 \operatorname{anos}^{(7)}$.

Entre 1996 e 2006, a média de filhos por mulher diminuiu de 2,5 para 1,8. Nesse período, adolescentes mais jovens, com menos de 14 anos, contribuíram com 26.752 (0,9\%) filhos vivos, enquanto aquelas mulheres entre 15 e 19 anos registraram 634.385 nascidos vivos $(20,9 \%)^{(8)}$. Em 2006, segundo dados do Sistema de Informações Hospitalares do Sistema Único de Saúde (SIH/SUS), gravidez, parto e puerpério responderam por $68,5 \%$ na proporção de internações hospitalares por grupos de causas em mulheres na faixa etária de dez a 19 anos.

O Sistema de Informação da Atenção Básica (SIAB) do Estado do Ceará, em 2000, mostrou 24\% de gestantes cadastradas menores de 20 anos. Em 2005, o SIAB registrou 268 nascimentos de filhos de mães com idade até 14 anos $(0,7 \%)$ e 7.422 entre 15 e 19 anos $(19,1 \%)$, valores similares aos do cenário nacional. Em 2006, gravidez, parto e puerpério responderam por $65,3 \%$ das internações hospitalares por grupos de causas, em mulheres na faixa etária de $1 \mathrm{dez}$ a 19 anos do Ceará.

A idade materna não pode, isoladamente, ser considerada responsável por eventos adversos na gravidez em adolescentes $^{(2,9)}$. Estudos apontam para associações significativas entre idade materna e complicações perinatais como eclâmpsia, hipertensão, partos cirúrgicos, lesões perineais, rotura prematura das membranas, desproporção cefalopélvica, sofrimento fetal agudo, aborto, infecção urinária, anemia, apresentação pélvica, placenta prévia, prematuridade, baixo peso, infecções e mortalidade neonatal precoce $e^{(4,10-17)}$. O risco gestacional na adolescência apresenta determinantes não só biológicos, mas principalmente psicológicos, culturais e socioeconômicos ${ }^{(2,5,9,10)}$.

O maior impacto da gravidez na adolescência é psicossocial $^{(2,18)}$ e se traduz em deserção escolar e baixa escolaridade, maior número de filhos, desemprego mais frequente, fracasso na relação com o parceiro (maior risco de separação, divórcio e abandono) e menor probabilidade de alcançar bons salários por toda a vida. Segundo dados da Organização Pan-Americana da Saúde, a gravidez na adolescência contribui para perpetuar o ciclo da pobreza e para a "feminização" da miséria ${ }^{(19)}$. A pobreza pode exacerbar, em alguns indivíduos, tais riscos ${ }^{(1,15,20-23)}$

Este estudo objetivou verificar a associação entre gestação na adolescência (dez a 19 anos) e baixo peso ao nascer, em maternidade pública de Fortaleza (CE), no Nordeste brasileiro.

\section{Método}

Estudo transversal realizado na Maternidade Escola Assis Chateaubriand (MEAC), instituição de referência terciária estadual, que desenvolve atividades de assistência, ensino, pesquisa e extensão, integrante do campus da Faculdade de Medicina da Universidade Federal do Ceará (UFC).

Por intermédio de sorteio aleatório simples, foram incluídas mulheres que tiveram seus filhos na MEAC da UFC no período de junho de 2000 a junho de 2001. Para estimativa do tamanho amostral, utilizou-se o aplicativo Epi-Info 6.0, com os seguintes parâmetros: intervalo de confiança de $95 \%$, poder de $80 \%$, prevalência estimada de recém-nascidos de baixo peso em adolescentes de 19\%, prevalência estimada de recém-nascidos de baixo peso em não-adolescentes de $10 \%$, Odds Ratio de 2,0. O tamanho da amostra calculada foi de 265 em cada grupo de mães e recém-nascidos, totalizando 
530 duplas. Foram utilizados como critérios de exclusão da pesquisa: mães adolescentes com gestações múltiplas, com perdas fetais, gravemente enfermas, aquelas que recusaram participar da pesquisa e os registros de partos sem menção do peso ao nascer.

Os dados foram coletados entre junho de 2000 e junho de 2001. As adolescentes (dez a 19 anos) e mulheres adultas (20 anos ou mais) foram identificadas no livro de registros da Maternidade Escola, no qual consta o nome, idade, diagnóstico de entrada da paciente, entre outras informações. Após identificar as pacientes e proceder ao sorteio simples, realizou-se uma entrevista com cada mãe, explicando-se natureza da pesquisa para obter seu consentimento informado. Foram entrevistadas 213 adolescentes e 340 mulheres adultas, valores inferiores aos estimados por problemas na logística do estudo.

A entrevista foi realizada nas primeiras 24 horas após o parto, com formulário estruturado e previamente testado. Paralelamente, os alunos pesquisadores de campo consultaram os prontuários médicos obstétricos e dos recém-nascidos para complementar os dados clínicos das pacientes e de seus filhos. Os pediatras da maternidade obtiveram as medidas antropométricas dos neonatos logo após o nascimento. A avaliação da idade gestacional foi obtida por meio de exame físico realizado pelos pediatras da instituição, preferentemente nas primeiras 24 horas e eventualmente nas primeiras 48 horas de vida, utilizando-se o método de Capurro ${ }^{(24)}$. A qualidade da assistência pré-natal foi avaliada em função do mês de início do pré-natal e do número de consultas realizadas.

A variável dependente do estudo foi ocorrência de baixo peso ao nascer (inferior ou igual a $2500 \mathrm{~g}$ ). A análise preliminar descritiva permitiu construir um perfil das gestantes e a escolha de variáveis de importância para a associação estudada. Com a finalidade de afastar a possibilidade de a diferença detectada na ocorrência de baixo peso ao nascer entre os grupos da amostra se dever à prematuridade, foi realizada a análise univariada. A regressão logística foi utilizada como procedimento para ajustar as variáveis de confusão. Por sua importância do ponto de vista clínicoepidemiológico na determinação do baixo peso ao nascer, tal como descrito na literatura revisada, foram selecionadas as seguintes variáveis como prováveis fatores de confusão: escolaridade da mãe ( $<5$ e $\geq 5$ anos de estudo), condição nutricional no início da gestação (índice de massa corpórea $[\mathrm{IMC}\}<18, \mathrm{IMC} \geq 18$ ), presença ou ausência do companheiro, realização de pré-natal de qualidade (mínimo de seis consultas pré-natal e início no primeiro trimestre), primiparidade (categorizada como gestante primípara e multípara) e prematuridade (categorizado como $<37$ e $\geq 37$ semanas). Foram considerados como potenciais fatores de confusão as variáveis selecionadas que alteraram em 10\% (para mais ou para menos) os valores da medida de Odds Ratio.

Todos os questionários foram processados em base eletrônica por intermédio do programa estatístico Epi-Info 6.0. A limpeza dos dados foi realizada por análise de consistência, buscando-se erros de amplitude. Os erros encontrados foram corrigidos no banco de dados, recorrendo-se ao arquivo da MEAC, para eventual recuperação dos prontuários e correção de registros duvidosos. Os dados foram analisados utilizando-se os aplicativos Epi-Info 6.0 e STATA 7.0.

Os referenciais da Bioética, preconizados na resolução n. ${ }^{\circ}$ 196/96 do Conselho Nacional de Saúde (CNS), foram considerados neste estudo, tendo sido obtida aprovação do Comitê de Ética em Pesquisa da MEAC da UFC.

\section{Resultados}

Foram selecionadas 539 duplas (mães e seus respectivos recém-nascidos), sendo 331 (61,4\%) mulheres com 20 anos ou mais e 208 (38,5\%) mulheres com idade <20 anos. A média do peso ao nascer foi $3067 \mathrm{~g}$ (desvio padrão $[\mathrm{DP}]=681 \mathrm{~g}$ ) para as duplas. Os recém-nascidos de mães adolescentes e adultas apresentaram média de peso de $2951 \pm 644 \mathrm{~g}$ e $3139 \pm 695 \mathrm{~g}$, respectivamente.

Como mostrado na Tabela 1, ocorreram 52 (25\%) recémnascidos com peso inferior a $2500 \mathrm{~g}$ nas gestantes adolescentes e $56(16,9 \%)$ nas adultas (OR 1,64; IC95\% 1,05-2,56). A ocorrência de prematuridade na população analisada foi de $102(18,9 \%)$ casos, com 50 (24\%) entre as adolescentes e 52 $(15,7 \%)$ entre as adultas (OR 1,58; IC95\% 1,00-2,51). Na Tabela 2, encontra-se a ocorrência de baixo peso ao nascer $(\leq 2500 \mathrm{~g})$ e peso insuficiente ao nascer $(>2500 \mathrm{e}<3000 \mathrm{~g})$ nos conceptos das mulheres estratificadas em quatro grupos, de acordo com sua idade: adolescentes até 16 anos; adolescentes de 17 a 19 anos; adultas de 20 a 34 anos; adultas com 35 anos de idade ou mais.

A frequência ao serviço de pré-natal foi bastante alta neste estudo: apenas $5(2,4 \%)$ adolescentes e $4(1,2 \%)$ adultas não o realizaram. $\mathrm{O}$ número médio de consultas durante o atendimento pré-natal foi de cinco entre as adolescentes e de seis entre as adultas. Observa-se que $269(48,6 \%)$ gestantes compareceram a, pelo menos, seis consultas de pré-natal, sendo essa característica notada em $90(42,3 \%)$ adolescentes e $179(52,6 \%)$ mulheres adultas. A idade gestacional média 
Tabela 1 - Distribuição dos recém-nascidos em relação à idade gestacional

\begin{tabular}{lrrrrrr}
\hline & \multicolumn{2}{c}{ RN mãe adolescente } & \multicolumn{2}{c}{ RN mãe adulta } & \multicolumn{2}{c}{ Total } \\
\cline { 2 - 7 } & \multicolumn{1}{c}{$\mathbf{n}$} & \multicolumn{1}{c}{$\boldsymbol{n}$} & \multicolumn{1}{c}{$\boldsymbol{\%}$} & $\mathbf{n}$ & \multicolumn{1}{c}{$\%$} \\
\hline Adequados para a IG & 150 & 72,1 & 247 & 74,6 & 397 & 73,6 \\
Pequenos para a IG & 50 & 24,0 & 52 & 15,7 & 102 & 18,9 \\
Grandes para a IG & 8 & 3,9 & 32 & 9,7 & 40 & 7,5 \\
Total & $\mathbf{2 0 8}$ & $\mathbf{1 0 0 , 0}$ & $\mathbf{3 3 1}$ & $\mathbf{1 0 0 , 0}$ & $\mathbf{5 3 9}$ & $\mathbf{1 0 0 , 0}$ \\
\hline
\end{tabular}

Tabela 2 - Frequência de baixo peso e peso insuficiente ao nascer de acordo com a idade materna

\begin{tabular}{lrrrrrr}
\hline \multirow{2}{*}{ Idade materna (anos) } & \multicolumn{2}{c}{ Baixo peso } & \multicolumn{2}{c}{ Peso insuficiente } & \multicolumn{2}{c}{ Total } \\
\cline { 2 - 7 } & $\mathbf{n}$ & $\mathbf{\%}$ & $\mathbf{n}$ & $\mathbf{\%}$ & $\mathbf{n}$ & \multicolumn{1}{c}{$\%$} \\
\hline $10-16$ & 20 & 38,4 & 15 & 32,6 & 35 & 35,7 \\
$17-19$ & 32 & 61,6 & 31 & 67,4 & 63 & 64,3 \\
Total de adolescentes & 52 & 100,0 & 46 & 100,0 & 98 & 100,0 \\
$20-34$ & 49 & 87,5 & 52 & 85,2 & 94 & 85,4 \\
35 ou mais & 7 & 12,9 & 9 & 14,8 & 16 & 14,5 \\
Total de adultas & 56 & 100,0 & 61 & 100,0 & 110 & 100,0 \\
Total & $\mathbf{1 0 8}$ & & $\mathbf{1 0 7}$ & & $\mathbf{2 0 8}$ & \\
\hline
\end{tabular}

de início do pré-natal foi de 12 semanas entre as adolescentes e de 14 semanas entre as adultas. Constatou-se que $207(37,4 \%)$ gestantes iniciaram o pré-natal no primeiro trimestre: $74(34,7 \%)$ adolescentes e $133(39,1 \%)$ adultas. Utilizando-se o critério de assistência pré-natal de qualidade mínima (início no primeiro trimestre gestacional e, pelo menos, seis consultas), a ocorrência de baixo peso ao nascer associou-se com atendimento pré-natal adequado: entre as pacientes que realizaram pré-natal adequado, a ocorrência de baixo peso foi de $12,3 \%$, enquanto $22,1 \%$ apresentavam peso abaixo de $2500 \mathrm{~g}$ no grupo cuja assistência foi inadequada.

Foi utilizado o IMC para classificar as pacientes quanto ao estado nutricional no início da gravidez. Foi selecionado o grupo de mulheres que apresentou IMC $<18$ como indicativo de algum grau de desnutrição. Na população avaliada, 64 mulheres atingiram o critério proposto para desnutrição, das quais $49(76,6 \%)$ tiveram bebês com peso > 2500g e $15(23,4 \%)$, bebês com baixo peso. Nesse grupo com IMC $<18,37(57,8 \%)$ eram adolescentes e $27(42,2 \%)$, adultas. Entre as adultas e as adolescentes com IMC baixo, 5 (18,5\%) e 10 (27\%) tiveram recém-nascidos de baixo peso, respectivamente.

Em relação ao estado civil, $42(20,1 \%)$ mães adolescentes estavam casadas, $110(52,8 \%)$ encontravam-se em união consensual, $2(0,9 \%)$ estavam separadas e $24(11,5 \%)$, solteiras sem companheiro. Situação diversa quando comparadas às mães com 20 anos ou mais: $122(36,8 \%)$ casadas, $153(46,2 \%)$ em união consensual, $5(1,5 \%)$ separadas e 32 $(9,6 \%)$ solteiras sem companheiro.
Utilizando-se a análise univariada e tendo-se como parâmetro a associação encontrada entre a ocorrência de baixo peso ao nascer e a baixa idade materna, selecionaram-se as variáveis: prematuridade, primiparidade, escolaridade, assistência pré-natal de qualidade e presença do companheiro, no intuito de esclarecer se outras condições contribuíam para associação independente dessas variáveis e recém-nascido de baixo peso de mães adolescentes (Tabela 3). Esta análise mostrou que as variáveis escolhidas não se comportaram como fatores de confusão.

$\mathrm{Na}$ análise de regressão logística, observou-se que a prematuridade configurou-se como a maior causa da ocorrência de baixo peso ao nascer e que a mesma exerceu influência tão marcante no grupo de variáveis, que todas as outras perderam significância. Diante disso, foi construído um modelo em que essa variável não estivesse presente. Nesta casuística, a associação encontrada na análise univariada entre baixo peso e mãe adolescente não se manteve na análise multivariada (Tabela 4).

\section{Discussão}

A proporção de baixo peso ao nascer tem sido considerada um marcador da saúde perinatal e de qualidade de vida, guardando uma relação evidente com os níveis de morbimortalidade infantil, principalmente nos países em desenvolvimento ${ }^{(6,25-27)}$. Alguns achados na literatura sustentam que o peso do recém-nascido aumenta à medida que 
aumenta a idade da mãe e que a incidência de baixo peso ao nascer é maior em mães adolescentes ${ }^{(2,22,23,28-30)}$. No entanto, outros estudos consideram contraditória a influência da idade materna no peso ao nascer, motivando a busca por respostas que esclareçam os verdadeiros preditores de baixo peso ao nascer.

Os resultados da análise multivariada desta casuística (em que se optou por retirar a prematuridade do modelo) mostraram que a gravidez na adolescência não se associa ao baixo peso ao nascer, quando ajustada por outras variáveis que influenciam esse desfecho. De maneira similar, Mariotoni e Barros Filho ${ }^{(31)}$, em estudo de caso controle conduzido na Maternidade de Campinas, concluíram que a gravidez na adolescência não representou maior risco para a ocorrência de baixo peso ao nascer na população estudada. Outra pesquisa, utilizando variáveis das declarações de nascido vivo, mostrou que, nas gestações a termo de mães com maior idade (>34 anos), houve maior chance de ocorrência de baixo peso ao nascer ${ }^{(32)}$. Inquérito conduzido em Ribeirão Preto (SP) concluiu que, quando o risco para o nascimento a termo de baixo peso foi ajustado para algumas variáveis relacionadas com o comportamento reprodutivo, como raça, escolaridade, paridade, situação conjugal e cuidados pré-natais, houve menor risco para mães adolescentes, e tal risco aumentou com o avanço da idade da mãe, sugerindo que a chance elevada de baixo peso ao nascer em adolescentes grávidas é reflexo de uma aglomeração de outros fatores de risco, presentes nessa faixa etária ${ }^{(33)}$.

Vários estudos encontraram relação entre o ganho de peso da gestante e o peso do recém-nascido. Um estudo de coorte retrospectiva em instituição privada de alta condição socioeconômica de nível terciário na cidade de São Paulo concluiu que gestações com ganho ponderal $\leq 10 \mathrm{~kg}$ têm risco maior de resultar em recém-nascidos de baixo peso, e gestações com ganhos ponderais $<12 \mathrm{~kg}$ têm risco maior de resultar em recém-nascidos com peso insuficiente ${ }^{(25)}$.

$\mathrm{Na}$ América Latina, informações da "Investigação Colaborativa Latino-Americana de Baixo Peso ao Nascer", realizada em 59 hospitais públicos de 11 países, indicam que aproximadamente 40-50\% dos recém-nascidos são pequenos para a idade gestacional (PIG), isto é, apresentam restrição do crescimento intrauterino e iniciam a vida já em desvantagem, com algum grau de desnutrição. Tal fato acarreta risco aumentado de adoecer e morrer durante o primeiro ano de vida ${ }^{(34)}$. Em Fortaleza, em estudo realizado em 17 maternidades onde aconteceram 40.712 partos no ano de 1995, a frequência de baixo peso entre os filhos de gestantes
Tabela 3 - Resultados da análise bivariada segundo a relação entre mães adolescentes e baixo peso ao nascer

\begin{tabular}{lcccc}
\hline & OR & $\begin{array}{c}\text { OR } \\
\text { ajustada }\end{array}$ & $\begin{array}{c}\text { Valor } \\
\boldsymbol{p}\end{array}$ & $\begin{array}{c}\text { Provável } \\
\text { confusão }\end{array}$ \\
\hline Prematuridade & 1,64 & 1,48 & 0,14 & Não \\
Primiparidade & 1,64 & 1,70 & 0,18 & Não \\
Escolaridade & 1,64 & 1,72 & 0,33 & Não \\
Pré-natal & 1,64 & 1,57 & 0,51 & Não \\
Companheiro & 1,64 & 1,56 & 0,96 & Não \\
\hline
\end{tabular}

OR: Odds Ratio

Tabela 4 - Resultados da análise multivariada para a variável dependente baixo peso ao nascer

\begin{tabular}{lccc}
\hline & OR & IC95\% & $\begin{array}{c}\text { Valor } \\
\boldsymbol{p}\end{array}$ \\
\hline Adolescência & 1,30 & $0,77-2,21$ & 0,321 \\
Desnutrição materna & 1,45 & $0,83-2,54$ & 0,183 \\
Fumante & 1,50 & $0,67-3,35$ & 0,315 \\
Analfabetismo & 1,27 & $0,66-2,41$ & 0,463 \\
Sem companheiro & 1,03 & $0,49-2,14$ & 0,929 \\
Multípara & 1,02 & $0,59-1,74$ & 0,930 \\
Pré-natal adequado & 0,54 & $0,29-1,00$ & 0,054 \\
\hline
\end{tabular}

OR: Odds Ratio; IC95\%: intervalo de confiança de 95\%

adolescentes foi $9,5 \%$, sendo $12,2 \%$ no grupo de dez a 14 anos e 9,4\% entre as maiores de 14 anos, em um cenário no qual a taxa de baixo peso em recém-nascidos de mulheres adultas foi $6,8 \%{ }^{(35)}$.

Estudo de coorte retrospectivo realizado em Glasgow, que analisou os nascimentos ocorridos entre 1992 a 1998 , comparando-se pacientes de 15-19 anos e de 20-29 anos após a primeira e a segunda gestações, evidenciou associação importante de óbito fetal e prematuridade, constituindo risco três vezes maior desses eventos em adolescentes na segunda gestação $^{(36)}$. É de extremo interesse observar a presença, neste estudo, de adolescentes na segunda e mesmo na terceira gestação: 42 jovens eram secundíparas e 12 estavam na terceira gravidez, "desafiando" a situação de duplo anabolismo e reincidindo nessa condição.

Os resultados apresentados neste estudo sugerem que a idade materna não está associada isoladamente com o baixo peso ao nascer. As pacientes com idade menor que 14 anos, aquelas com idade ginecológica abaixo de dois anos e as adolescentes multíparas merecem estudo à parte, havendo indícios de que nesses grupos as repercussões da gravidez podem se tornar desfavoráveis para mãe e filho. 


\section{Referências bibliográficas}

1. Brasil - Pesquisa Nacional de Demografia e Saúde da Criança e da Mulher (PNDS) [homepage on the Internet]. Fecundidade e intenções reprodutivas das mulheres. Brasília: Ministério da Saúde [cited 2009 Jan 05]. Available from: http://bvsms.saude.gov.br/bvs/pnds/fecundidade.php

2. Cabral MG. Conseqüências da gravidez na adolescência: riscos para a saúde da mãe e do recém-nascido [tese de mestrado]. Recife: UFP; 1997.

3. Berquó $\mathrm{E}$, Cavenaghi $\mathrm{S}$ [homepage on the Internet]. Mapeamento sócioeconômico e demográfico dos regimes de fecundidade no Brasil e sua variação entre 1991 e 2000 [cited 2010 Dec 09]. Available from: http://www.nepo.unicamp. br/aulas/2010/zm/graduacao/MapeamentoSocioeconomico.pdf.

4. Goldani MZ, Bettiol H, Barbieri MA, Tomkins A. Maternal age, social changes, and pregnancy outcome in Ribeirão Preto, southeast Brazil, in 1978-79 and 1994. Cad Saude Publica 2000;16:1041-7.

5. Magalhães ML. Aspectos da gravidez na adolescência em maternidade escola de Fortaleza [tese de mestrado]. São Paulo: Unifesp; 2004.

6. Gama SG, Szwarcwald CL, Leal MC, Theme Filha MM. The pregnancy during adolescence as a risk factor for low birth weight, Brazil. Rev Saude Publica 2001;35:74-80.

7. Brasil - Ministério da Saúde. Datasus [homepage on the Internet]. Informações de saúde . Estatísticas vitais: mortalidade e nascidos vivos [cited 2010 Sep 29]. Available from: http://tabnet.datasus.gov.br/cgi/deftohtm.exe?sinasc/cnv/nvuf.def

8. Brasil - Instituto Brasileiro de Geografia e Estatística. Indicadores Sociais Brasileiros. Rio de Janeiro: IBGE; 2006.

9. Mathias L, Nestarez JE, Kanas M, Neme B. Gravidez na adolescência: IV. Idade limite de risco reprodutivo entre adolescentes. J Bras Ginecol 1985;95:141-3.

10. Chen XK, Wen SW, Fleming N, Demissie K, Rhoads GG, Walker M. Teenage pregnancy and adverse birth outcomes: a large population based retrospective cohort study. Int J Epidemiol 2007;36:368-73.

11. Creasy RK, Parer JT. Prenatal care and diagnosis. In: Rudolph AM, Hoffman Jl. Pediatrics. $17^{\text {a }}$ ed. Norwalk: Appleton-Century-Crofts; 1982. p. 105-17.

12. Goonewardene IM, Deeyagaha Waduge RP. Adverse effects of teenage pregnancy. Ceylon Med J 2005;50:116-20.

13. Magalhães ML, Furtado FM, Nogueira MB, Carvalho FH, Almeida FM, Mattar $\mathrm{R}$ et al. Precocious and late pregnancy in adolescents - is there a difference comparing the obstetric risks? Rev Bras Ginecol Obstet 2006;28:446-52.

14. McAnarney ER, Greydanus DE. Adolescência. In: Kempe CH, Silver HK, O'Brien D. Pediatria - diagnóstico e tratamento. $8^{\mathrm{a}}$ ed. Rio de Janeiro: Guanabara; 1986. p. 176-205.

15. Pavlova-Greenfield T, Sutija VG, Gudavalli M. Adolescent pregnancy: positive perinatal outcome at a community hospital. J Perinat Med 2000;28:443-6.

16. Ribeiro ER, Barbieri MA, Bettiol H, Silva AA. Comparison between two cohorts of adolescent mothers in Southeastern Brazil. Rev Saude Publica 2000; $34: 136-42$

17. Sabroza AR, Leal MC, Souza Júnior PR, Gama SG. Some emotional repercussions of adolescent pregnancy in Rio de Janeiro, Brazil (1999-2001). Cad Saude Publica 2004;20:130-7.

18. Maia Filho NL, Mathias L, Tedesco RP, Cesareo MA, Porta RM. Gravidez entre adolescentes precoces: um evitável problema social. J Bras Ginecol 1994;104:363-7.
19. Romero TC. Gravidez en la adolescencia. Ginecol Obstet Mexico 1992;60:291-5.

20. Chen XK, Wen SW, Fleming N, Yang Q, Walker MC. Increased risks of neonatal and postneonatal mortality associated with teenage pregnancy had different explanations. J Clin Epidemiol 2008;61:688-94.

21. Gigante DP, Barros FC, Veleda R, Gonçalves H, Horta BL, Victora CG. Maternity and paternity in the Pelotas birth cohort from 1982 to 2004-5, Southern Brazil. Rev Saude Publica 2008;42 (Supl 2):42-50.

22. Keskinoglu P, Bilgic N, Picakciefe M, Giray H, Karakus N, Gunay T. Perinatal outcomes and risk factors of Turkish adolescent mothers. J Pediatr Adolesc Gynecol 2007;20:19-24.

23. Kurauchi AT, Roteli-Martins CM, Aquino MM. Impacto da gravidez na adolescência e resultados perinatais: revisão da literatura. Femina 2003;31:669-71.

24. Capurro H, Konichezky S, Fonseca D, Caldeyro-Barcia R. A simplified method for diagnosis of gestational age in the newborn infant. $\mathrm{J}$ Pediatr 1978;93:120-2.

25. Lizo CL, Azevedo-Lizo Z, Aronson E, Segre CA. Relationship between materna weight gain and birthweight. J Pediatr (Rio J) 1998;74:114-8.

26. Mahavarkar SH, Madhu CK, Mule VD. A comparative study of teenage pregnancy. J Obstet Gynaecol 2008;28:604-7.

27. Kassar SB, Gurgel RQ, Albuquerque MF, Barbieri MA, Lima MC. Peso ao nascer de recém-nascidos de mães adolescentes comparados com o de puérperas adultas jovens. Rev Bras Saude Matern Infant 2005; 5:293-9.

28. Guerra AF, Heyde ME, Mulinari RA. Impacto do estado nutricional no peso ao nascer de recém-nascidos de gestantes adolescentes. Rev Bras Ginecol Obstet 2007;29:126-33.

29. Rocha RC, Souza E, Guazzelli CA, Chambô Filho A, Soares EP, Nogueira ES. Prematuridade e baixo peso entre recém-nascidos de adolescentes primíparas. Rev Bras Ginecol Obstet 2006;28:530-5.

30. Salinas PH, Erazo BM, Pastene SC, Reyes PA, Catalán MJ, Carmona GS. Factores de riesgo asociados al bajo peso al nacer. Rev Chil Salud Publica 2004;8:78-83.

31. Mariotoni GG, Barros Filho AA. A gravidez na adolescência é fator de risco para o baixo peso ao nascer? J Pediatr (Rio J) 1998;74:107-13.

32. Carniel EF, Zanolli ML, Antonio MA, Morcillo AM. Determinantes do baixo peso ao nascer a partir das Declarações de Nascidos Vivos. Rev Bras Epidemiol 2008;11:169-79.

33. Silva AA, Gomes UA, Bettiol H, Dal Bo CM, Mucillo G, Barbieri MA. Associação entre idade, classe social e hábito de fumar maternos com peso ao nascer. Rev Saude Publica 1992;26:150-4.

34. Puffer RR, Serrano CV. Características del peso al nascer. Washington (DC): OMS; 1988.

35. Leite AJ. Mortalidade perinatal e aspectos da qualidade da atenção à saúde no município de Fortaleza, Ceará, 1995 [tese de mestrado]. São Paulo: UNIFESP; 1996.

36. Smith GC, Pell JP. Teenage pregnancy and risk of adverse perinatal outcomes associated with first and second births: population based retrospective cohort study. BMJ 2001;323:476. 